\title{
Tintin et le futur antérieur : de la conjecture à l'anaphore
}

\author{
Jean-Paul Meyer \\ Université de Strasbourg, Laboratoire LiLPa (UR 1339) \\ jpmeyer@unistra.fr
}

\section{Introduction}

Le futur antérieur conjectural est un temps étrange. Formé pour émettre une supposition sur un fait passé, il est construit comme un passé composé dont l'auxiliaire est au futur. En cela, il permet de dédoubler le moment d'énonciation et de projeter dans le futur le moment présumé de la fin de la supposition ${ }^{2}$.

Par rapport au futur antérieur temporel « classique »

Dans quelques heures, le " Ramona » aura disparu corps et biens...

(CS51D4, Rastapopoulos ${ }^{3}$ )

où c'est le point de référence qui est décalé dans le futur (et où par conséquent la temporalité à venir est décisive), le futur antérieur conjectural

Nestor se sera fait du mauvais sang pour d'innocentes gamineries...

(CS61D4, Haddock)

reporte à plus tard (mais sans aucunement le situer dans le temps ${ }^{4}$ ) le moment où l'évènement, qui est déjà accompli, pourra être dit accompli. Dans le futur antérieur conjectural, l'advenu prédomine et la temporalité ne joue qu'un très petit rôle.

Le report sine die de la fin de la supposition fait donc de ce temps verbal un précieux vecteur d'inférence ${ }^{5}$ :

Je parie que c'est Tintin qui a fait le coup : il se sera enfui quand il nous aura entendus.

(IN51B1, Müller)

Est-ce pour cela qu'il est si massivement représenté dans les Aventures de Tintin? Avec près de cinquante occurrences au long des 23 albums de la série, dont quelques emplois très inattendus (voir le corpus complet en annexe 2 infra), le futur antérieur conjectural peut être considéré comme une constante de l'œuvre. La conjecture est à n'en pas douter une modalité d'action qui convient au personnage de Tintin, à ses aventures et ses enquêtes. Ne faut-il pas aussi voir dans cette fréquence la marque d'un fonctionnement particulier du récit iconotextuel, voire pour Hergé la manière de délivrer un message implicite?

Nous aurons à répondre à ces questions, après avoir analysé le corpus que forment les occurrences recensées dans l'ensemble des albums, puis répertorié les fonctions assignées au futur antérieur dans les phylactères et les cases. En examinant plus longuement certains contextes d'emploi qui permettent d'expliquer le rôle référentiel du futur antérieur conjectural, il sera finalement possible de se demander dans quelle mesure une anaphore peut être conjecturale.

\section{Le temps de la reconstitution}

Le futur antérieur conjectural est un emploi modal du futur antérieur. Si du point de vue temporel il est généralement associé au passé composé , sa valeur le tourne du côté de la conjecture, c'est-à-dire de l'hypothèse non avérée, de la supposition ${ }^{7}$. L'appellation futur antérieur conjectural (désormais $\mathrm{FA}_{\mathrm{C}}$ ) est d'ailleurs reconnue par la plupart des auteurs ${ }^{8}$, même si certains préfèrent le terme épistémique, qui rappelle que ce futur est souvent en concurrence avec la modalité épistémique du verbe «devoir» au passé composé : Il sera venu hier / Il a dû venir hier?. 
Plusieurs grammaires de référence expliquent la valeur conjecturale du futur antérieur en la dérivant du futur de supposition. C'est le cas de Wagner \& Pinchon (1978 : 357) ou de Riegel et al. (2009: 553), ainsi que de Grevisse \& Goosse (2007: 1097) pour qui la tournure sert à «exprimer l'explication probable d'un fait présent ». Un problème se pose toutefois, dans cette dérivation, à cause de la substitution opérée entre fait présent (futur conjectural) et fait passé (futur antérieur conjectural). S'il est généralement admis que le futur antérieur puisse «exprimer une supposition qui porte sur le passé » (Riegel et al., 2009: 554), c'est parce que «l'ignorance [du] fait passé sera dissipée dans l'avenir » (Martin, 1992: 141). Or cette dernière condition n'est que très rarement réalisée : il lui suffit généralement d'être présumée, voire garantie «sur parole», comme nous le verrons dans le corpus étudié.

Le $\mathrm{FA}_{\mathrm{C}}$, en tant que présomption posée sur le passé, fonde les énoncés qui cherchent à expliquer après coup un enchainement de faits, sur le modèle de la reconstitution, du soupçon, ou encore de la scène finale de l'enquête.

Mais alors, j’y songe, le petit papier... se sera envolé lorsque je suis entré, la première fois, dans mon bureau, pour y prendre ma loupe !...

(CO7B2-B3, Tintin)

Dans ce type de discours, le passage de la nature conjecturale-valeur habituelle de ce type d'énoncés - à la nature explicative dépend du statut de celui qui emploie le $\mathrm{FA}_{\mathrm{C}}$. La légitimité de l'explication, son acceptabilité en quelque sorte, est proportionnelle à l'autorité de l'énonciateur ${ }^{10}$. Plus cette autorité est grande, moins la conjecture est mise en doute, comme en témoigne cet exemple extrait de Jules Verne :

- En effet, dit James Starr, tout s'explique. Un hasard a révélé à Silfax l'existence
du nouveau gisement. Dans son égoüsme de fou, il aura voulu s'en constituer le
défenseur. Vivant dans la houillère, la parcourant nuit et jour, il aura surpris votre
secret, Simon, et su que vous me demandiez en toute hâte au cottage. De là, cette
lettre contradictoire de la vôtre ; de là, après mon arrivée, le bloc de pierre lancé
contre Harry et les échelles détruites du puits Yarow [...J.
- Vous venez de raconter les choses comme elles ont évidemment dû se passer,
monsieur James, répondit Simon Ford.

(Jules Verne, Les Indes-Noires, chap. 20)

On voit dans ce type d'énoncé combien le $\mathrm{FA}_{\mathrm{C}}$ prend une valeur performative, se proclamant à la fois conjecture et explication, la seconde prenant progressivement la place de la première et rendant caduque l'attente d'une vérification à venir.

D'une façon générale, le $\mathrm{FA}_{\mathrm{C}}$ est employé par l'expert de la situation (l'enquêteur génial, l'ingénieur malin, le savant qui prévoit tout, etc.), ce qui dispense son entourage de la vérification éventuelle. Et lorsque l'on vérifie quand même, ce n'est que pour montrer combien l'expert avait raison, renforçant du même coup son autorité. On le voit: l'expert prouve que son explication n'est pas une conjecture en imposant le fait qu'il n'y a pas d'autre... explication. La position affirmée ainsi par ce locuteur particulier, son interprétation épistémique des faits en quelque sorte, relève d'une "représentation discursive » à visée stratégique (Haillet, 2007). Dans la narration fictionnelle, en particulier dans le récit de quête ou d'enquête, l'expert est celui qui a le pouvoir de transformer son «évaluation subjective de la réalité » (Gosselin, $2010: 325$ ), propre à la modalité épistémique, en un récit ${ }^{11}$.

La possibilité d'une «explication conjecturale » provient, on l'a montré, du processus inférentiel particulier actionné par le futur antérieur. Comme cela a souvent été dit (Dendale \& De Mulder, 1996 ; Desclés \& Guentchéva, 2001 ; Gosselin, 2010), le $\mathrm{FA}_{\mathrm{C}}$ repose sur une inférence abductive ${ }^{12}$, l'abduction étant ce mode de raisonnement qui prend pour point de départ une hypothèse tenue pour (ou imposée comme) plausible ${ }^{13}$. Dans une situation «classique »d'ignorance, il ne peut d'ailleurs guère y avoir d'autres hypothèses que celles reconnues comme plausibles. Lorsque la vérification ne peut être remise à plus tard, en particulier lorsqu'une explication est requise, c'est en général la stratégie communicative qui fait accepter une explication comme telle. Dans le récit fictionnel - on l'a vu dans l'extrait des Indes Noires ci-dessus, mais c'est évidemment la même chose dans Tintin - le statut particulier du locuteur (héros ou autorité en la matière) prévu dans le contrat narratif impose l'acceptabilité de l'explication. Que 
la situation soit réelle ou fictionnelle ne change rien au fait qu'à chaque fois, la plausibilité se transforme en vérité, tout au moins en énoncé tenu pour vrai ${ }^{14}$.

Dans un article consacré à l'expression de la conjecture en français, Bellahsène propose de considérer deux types de contextes d'ignorance dans lesquels le futur conjectural se manifeste : l'un permettant la vérification de l'hypothèse, et l'autre autorisant la supposition non vérifiable (2007: 256). Le récit de fiction ne respecte pas cette division des choses, et encore moins le récit iconotextuel, comme nous le verrons dans notre troisième partie. Les aventures et le personnage de Tintin constituent en effet un contexte du deuxième type, alors qu'elles mettent en scène des situations de communication courantes (celles que Bellahsène classe dans les contextes du premier type) et malgré la présence d'hypothèses vérifiables qui les accompagnent.

\section{Le futur antérieur dans les Aventures de Tintin}

On dénombre 71 occurrences de futur antérieur dans l'ensemble des aventures ${ }^{15}$, dont 23 seulement sont à valeur temporelle. Cela représente un total de 48 cas de $\mathrm{FA}_{\mathrm{C}}(67,6 \%)$, près de la moitié $(22$ occurrences exactement) étant énoncés par Tintin lui-même. Le caractère remarquable de ces chiffres provient de la surreprésentation du $\mathrm{FA}_{\mathrm{C}}$. Les vingt-trois futurs antérieurs temporels constituent un volume normal pour une œuvre narrative de cette ampleur, avec une fréquence moyenne d'une occurrence par album. En revanche, les $48 \mathrm{FA}_{\mathrm{C}}$ sont un échantillon « anormal », aussi bien dans leur rapport global (plus de $2 \mathrm{FA}_{\mathrm{C}}$ sur 3 futurs antérieurs) que dans leur fréquence moyenne (plus de 2 par album). Cela signifie que Hergé fait le choix de cette forme verbale avant tout pour sa valeur modale, et qu'elle devient pour lui, du point de vue formel, un outil stylistique et énonciatif de premier ordre ${ }^{16}$. Du point de vue thématique et diégétique, il n'est pas inutile de signaler qu'elle traduit chez Tintin une sorte de don, au service de sa puissance d'investigation ${ }^{17}$.

L'un des éléments qui corroborent l'argument du FA $\mathrm{F}_{\mathrm{C}}$ en tant qu'outil chez Hergé (c'est-à-dire comme procédé narratif conscient et non comme tic verbal) est le fait que la fréquence des occurrences chute brutalement à mi-parcours des aventures de Tintin, précisément après le cycle du trésor. L'installation à Moulinsart après la découverte du trésor de Rackham le rouge (à partir de l'aventure des 7 Boules de cristal) signifie la fin des enquêtes et des chasses, et donc la fin des $\mathrm{FA}_{\mathrm{C}}$ fréquents (ils ne réapparaitront que dans un sursaut provoqué par l'affaire des Bijoux de la Castafiore).

Un autre point remarquable est la présence ou non d'un marquage du degré de probabilité de la conjecture $^{18}$. Non seulement une forte proportion des cas de $\mathrm{FA}_{\mathrm{C}}$ se présente sans marquage, mais on peut ajouter, comme preuve supplémentaire de la puissance conjecturale de Tintin, que seuls trois des FA $_{C}$ qu'il produit sur l'ensemble des albums comportent un marqueur.

Ce marquage fonctionne comme une sorte d' «indice de confiance », que le locuteur applique à son affirmation afin de faire état du degré de doute dans lequel il tient son propre énoncé. Il se présente sous deux formes habituelles: l'une est d'ordre lexical, réalisée par un adverbe (sans doute, surement, probablement, etc.) ; l'autre est d'ordre syntaxique, et consiste généralement en un embrayeur (je suppose, à mon avis, etc.). Comparons les trois exemples suivants, typiques de leur catégorie.

Exemple de marqueur de probabilité de forme lexicale ( 7 cas sur $48 / 14,5 \%$ ) :

Ils seront probablement partis pour le Caire.

(CP5D2, Lettre du chef de la secte Kih-Oskh)

Exemple de marqueur de probabilité de forme syntaxique (4 cas sur 48 / 8,5\%) :

Je me demande si Tournesol sera venu nous attendre...

(AT3B4, Tintin)

Exemple d'absence de marquage de probabilité (37 cas sur 48 / 77\%) :

Ça, c'est le docteur qui s'en va : il sera venu pour le plâtre du capitaine...

(BC16D3, Tintin)

Trois remarques s'imposent à propos de l'indice de probabilité. On peut tout d'abord vérifier, chose que l'analyse pragmatique a montrée depuis longtemps, que le marqueur est un signe de doute. Que 
l'adverbe soit sans doute ou certainement n'y change rien : dès lors qu'un indice est présent, il signale la probabilité. Par conséquent, l'absence de marquage peut seule signaler la certitude. Du point de vue du locuteur, le fait d'énoncer un $\mathrm{FA}_{\mathrm{C}}$ sans indice de probabilité indique l'affirmation d'une certitude, ou tout au moins indique le refus de prendre en compte la probabilité d'une erreur.

On peut à partir de là expliquer un peu mieux pourquoi la quasi-totalité des occurrences recensées chez Tintin se présente sans aucun marquage. Il y a en effet deux cas où Tintin se permet de négliger (parfois outrageusement !) la probabilité : soit parce qu'il veut imposer son hypothèse (nous reviendrons plus loin sur ce point), soit parce qu'il sait, au moment d'exprimer une supposition, qu'elle n'en est pas une. Ce savoir lui vient de son statut de représentant du narrateur, de voix narrative procurée en quelque sorte, qui fait déteindre sur lui un peu de l'omniscience du locuteur invisible.

La troisième remarque que l'on peut faire à ce stade concerne les cas de $\mathrm{FA}_{\mathrm{C}}$ chez les autres personnages des aventures. Chez eux en effet les conjectures sont toujours hasardeuses, et presque toujours approximatives ou fausses. Mieux : c'est le plus souvent l'image qui, en contrepoint de leur énoncé conjectural, leur donne tort; l'effet de contradiction texte-image est, comme nous le voyons maintenant, l'une des fonctions assignées au $\mathrm{FA}_{\mathrm{C}}$.

\section{Les fonctions du FA dans les Aventures}

L'analyse des emplois du futur antérieur conjectural dans les albums montre que ce temps assure deux fonctions essentielles. D'une part, en vertu de la modalité épistémique qui le sous-tend, le $\mathrm{FA}_{\mathrm{C}}$ structure et distribue l'information contenue dans la case ; d'autre part, grâce à la chronologie particulière qu'il installe, le $\mathrm{FA}_{\mathrm{C}}$ organise la marche narrative à l'échelle de la séquence d'images.

\subsection{Statut épistémique de l'information}

Dans un récit en bande dessinée, la case est l'unité narrative minimale (Groensteen, 1999 : 31-32). Si cette idée est maintenant bien admise, il convient cependant d'ajouter que la case est à double titre une unité narrative : en tant qu'instant pris dans le continuum spatiotemporel de l'action, et en tant que scène constituant un maillon dans la chaine de représentation des évènements. Rappelons également que la BD, comme le théâtre et d'autres arts du spectacle, vit sous le régime de la double communication: le discours des personnages - protagonistes de la situation de communication figurée - constitue pour le lecteur-spectateur le récit qui lui est raconté ; la communication imitée apporte les savoirs nécessaires à l'intelligibilité de l'histoire. L'intérêt de ce dispositif est qu'il permet de différencier ce que savent les uns et les autres: les connaissances des personnages et celles du lecteur peuvent ainsi être mises en concurrence, voire en conflit, pour créer des effets de tension. Le procédé est bien connu au théâtre. Dans la bande dessinée cependant, en raison de la nature iconotextuelle de la case, les informations que le lecteur trouve dans la case se présentent à la fois sous forme picturale et sous forme verbale. L'image a donc son propre contenu informatif, sa propre valeur épistémique. En conséquence, la double communication est enrichie par une relation texte-image (RTI) qui à son tour met en parallèle les connaissances dont disposent respectivement le lecteur et les protagonistes de l'histoire.

On devine aisément le bénéfice que l'on peut tirer de cette tension particulière lorsque le savoir de tel ou tel personnage est présenté, par le biais du FA $\mathrm{F}_{\mathrm{C}}$, comme conjectural. Dans un grand nombre des $\mathrm{FA}_{\mathrm{C}}$ énoncés par les locuteurs au sein des aventures de Tintin, l'image contredit la valeur conjecturale de l'énoncé. Le procédé reprend un ressort courant de la RTI, celui du conflit affiché entre les deux vecteurs de la représentation, généralement utilisé comme effet comique. Les emplois du $\mathrm{FA}_{\mathrm{C}}$ dans Tintin relèvent davantage du conflit logique, même s'ils ne sont pas dénués d'humour: un protagoniste énonce une supposition mais dans le même temps l'image lui donne tort ${ }^{19}$. Le $\mathrm{FA}_{\mathrm{C}}$, temps de la modalité épistémique, peut alors servir à détruire la valeur épistémique de l'énoncé. Hergé utilise fréquemment le $\mathrm{FA}_{\mathrm{C}}$ dans cet esprit; associé à la RTI, il rend le savoir inégal, ce qui permet à la fois de créer des effets de double lecture, mais aussi de provoquer une réaction d'identification à Tintin (par connivence, inquiétude, admiration, etc.). Tintin est celui que l'image ne contredit pas, celui à qui l'image obéit en quelque sorte.

Hergé place systématiquement certains personnages en désavantage par rapport au lecteur, tout comme, a contrario, il avantage outrageusement le héros par rapport au narrataire. En analysant le corpus, 
nous pouvons remarquer que ce système fonctionne à trois niveaux; il permet de comparer le savoir (S) $\mathrm{du}$ lecteur $(\mathrm{L})$ à celui du protagoniste $(\mathrm{P})$ qui énonce la conjecture.

$-\mathrm{S}_{\mathrm{L}}=\mathrm{S}_{\mathrm{P}}$. Le lecteur n'en sait pas plus que le personnage ; ce dernier fait une conjecture que le lecteur ne peut pas vérifier immédiatement mais qui se confirme plus loin ; le $\mathrm{FA}_{\mathrm{C}}$ justifie donc par avance un élément de la narration. Exemple typique :

Le contact de l'eau l'aura ranimé.

\section{(TA13B2, Bobby Smiles)}

$-\mathrm{S}_{\mathrm{L}}>\mathrm{S}_{\mathrm{P}}$. Grâce à l'image, le lecteur en sait plus que le personnage ; ce dernier fait une conjecture dont le lecteur peut vérifier immédiatement la fausseté. Un cas typique de cette deuxième possibilité se trouve dans Le Lotus bleu. On y voit Tintin sortir sa tête d'un vase de Chine où il est caché, vase posé juste derrière des bandits qui déclarent :

\section{Je crois que Tintin ne viendra pas. Non, il se sera méfié...}

(LB55C2, Serviteur du Lotus Bleu)

$-\mathrm{S}_{\mathrm{L}}<\mathrm{S}_{\mathrm{P}}$. Le personnage (mais c'est alors presque toujours Tintin) en sait plus que le lecteur. Rien dans l'image (voire : aucune image) ne permet au lecteur de vérifier la conjecture, qui s'impose donc comme seul récit possible. Associé à l'image, ce type particulier d'inférence agit sur la narration en reconstituant le récit après coup. Les cas sont nombreux et font ressortir le statut tout à fait central de Tintin comme «créateur» de l'aventure dont il est lui-même le héros. Exemple typique :

\section{Oui, on l'aura fait disparaitre !}

(CO61C3, Tintin)

Cette typologie est certes sommaire et demande à être testée à plus grande échelle. Elle fait cependant ressortir la position particulière de Tintin, qui semble commander au récit grâce au pouvoir que lui donne sa «pratique »du $\mathrm{FA}_{\mathrm{C}}$. Dans Tintin, le futur antérieur conjectural n'a en effet pas seulement pour mission d'assigner les rôles dans la case ; il structure également la séquence narrative.

\subsection{Fonctions narratives du FA}

Dans le récit dessiné, la case, en tant que scène cadrée et située, devient le maillon d'une chaine narrative. «Écartelée entre son désir d'autonomie et son inscription dans le récit » (Peeters, 1998: 22), elle rejoint une unité supérieure, la séquence, structure complète et autosuffisante seule capable de raconter une histoire en images. Dans la séquence, la dimension conjecturale du futur antérieur est rejointe par sa dimension temporelle. En effet, bien que le $\mathrm{FA}_{\mathrm{C}}$ se caractérise moins par sa temporalité que par sa modalité, l'emploi de cette forme verbale dans une structure aussi marquée par le temps que l'est la séquence de cases ne peut manquer d'influer sur la marche du récit. Dans les Aventures de Tintin, les effets du $\mathrm{FA}_{\mathrm{C}}$ sur la diégèse apparaissent comme un système, associé à la relation texte-image pour donner à la séquence une dynamique particulière. Nous pouvons ainsi distinguer trois fonctions du futur antérieur conjectural : la fonction régressive, la fonction d'enchainement et la fonction progressive. Toutes trois se fondent sur la logique inférentielle du $\mathrm{FA}_{\mathrm{C}}$.

\subsubsection{La fonction régressive}

On connait la capacité de Tintin à remonter des conséquences aux causes. En général, elle se manifeste à une étape décisive de l'enquête qu'il est en train de mener, à partir d'une intuition géniale qui sert de point de départ, ou d'un eurêka qui va permettre de tout expliquer. Tout lecteur de Tintin connait ces moments.

Il est cependant d'autres cas, très fréquents également, où Tintin remonte des conséquences aux causes, c'est lorsqu'il s'agit d'expliquer un fait par un autre qui l'a produit. Ces situations apparaissent quand ce que montre la séquence de cases («ce qui se passe ») doit être expliqué au lecteur. Tintin le fait en vertu de son don d'inférence, c'est en quelque sorte sa mission à ce moment-là. On retrouve là une 
situation de type $\mathrm{S}_{\mathrm{L}}<\mathrm{S}_{\mathrm{P}}$ évoquée précédemment. Le $\mathrm{FA}_{\mathrm{C}}$ que Tintin emploie dans ce type de séquences a une fonction régressive au sens où la conjecture énonce la cause, et une valeur épistémique au sens où la conjecture a pour but de révéler au lecteur les informations qui lui sont nécessaires pour se hisser au niveau de connaissance du héros. Exemple parmi d'autres :

\title{
Le thé !... Il aura bu du thé qui était répandu à terre !...
}

$$
\text { (LB12B3, Tintin) }
$$

Revoyons la séquence : alors que Tintin s'apprête à prendre le thé, un inconnu lui tire dessus et brise sa tasse ; Tintin le poursuit mais sans succès, et à son retour dans l'appartement trouve Milou inconscient. Il ne sait pas (pas encore) que le thé contient un narcotique. Le raisonnement de Tintin est donc le suivant: Milou est inconscient parce qu'il a bu du thé (« il aura bu... »); ce thé contient donc un narcotique.

Comme d'autres héros d'aventures au statut plus ou moins démiurgique, ou comme d'autres enquêteurs exceptionnels tels Holmes et Poirot, Tintin est en mesure d'expliquer un fait ou de reconstituer l'intégralité d'une scène à partir de l'abduction de sa causalité. Cette faculté est essentielle à deux titres. D'une part elle qualifie le héros en le virilisant. Sa qualité virile s'exprime en effet à travers deux compétences aussi indissociables qu'insurpassables : le coup de poing et le coup de force. D'autre part elle garantit l'existence et la validité du récit ${ }^{20}$, le héros-enquêteur étant celui qui construit l'histoire en la (re)prenant à son début (c'est l'essence même du futur antérieur).

\begin{abstract}
Voilà comment je reconstitue l'affaire. [...] Dans une lettre, notre ami décrit ses travaux à Topolino. Cette lettre aura été surprise par le domestique de Topolino, un Bordure nommé Boris, qui alerte les services secrets de son pays. Mais l'espionnage syldave a vent, lui aussi, de l'invention et envoie à Moulinsart un de ses agents. Celui-ci tombe là sur un rival bordure, qui le blesse.
\end{abstract}

(AT28C1-C2, Tintin)

\subsubsection{La fonction d'enchainement}

L'enchainement est un type de RTI général et trivial dans la bande dessinée, largement étudié par plusieurs spécialistes (Fresnault-Deruelle, Peeters, Groensteen, etc.). Rappelons qu'en BD, le passage d'une case à la suivante ou d'une séquence à l'autre est le vecteur fondamental de la continuité narrative. Dans l'enchainement, cette continuité est assurée par la traduction intersémiotique (au sens que Jakobson donne à ce terme) de l'information : ce qui est représenté sous forme graphique dans telle case est repris (sémantiquement parlant) sous forme linguistique dans telle autre.

Avec le $\mathrm{FA}_{\mathrm{C}}$, cette RTI de facture très classique-son fonctionnement sémantique repose principalement sur le fait que les variables déictiques du texte sont saturées par des informations venant de la représentation graphique -, bénéficie d'une qualification et d'une valeur renouvelées. En voici un exemple caractéristique, dont il faut cependant préciser le contexte :

\section{Il aura de nouveau perdu connaissance...}

(ML58B4, Baxter)

La scène se passe en même temps dans la fusée au moment de son retour vers la terre, et dans le poste de contrôle au sol. En raison du manque d'oxygène, les occupants de la fusée sont évanouis, Tintin est seul à la manœuvre, mais il est plus en plus faible. Dans la case 58B3, précédant donc celle donnée en exemple, nous voyons Tintin évanoui sur son siège et appelé par radio depuis la terre. Autrement dit, l'évènement mis en scène - Tintin perdant connaissance - se déroule en deux « espaces » différents (la Terre, le ciel), mais avec une certaine simultanéité. Celle-ci fait de Baxter-le directeur des opérations au sol, qui conjecture ce qui s'est passé - un protagoniste aveugle, capable de donner une explication juste à ce que le lecteur sait déjà ${ }^{21}$.

Les exemples de ce type illustrent le phénomène de point de vue (Sthioul, 1998) lié aux temps verbaux. La conjecture que fait Baxter, raisonnablement fondée sur l'itération du phénomène et donc son caractère prévisible (« de nouveau »), témoigne du changement de point de vue sur l'évènement. Dans le récit en images, ce changement se traduit par un montage par «champ-contrechamp » au sein de la 
séquence de cases, qui permet au récit de passer tout de go du cadre d'action [fusée] au cadre d'action [poste de contrôle] sans rupture de la continuité narrative.

\subsubsection{La fonction progressive}

La relation de progression assurée par le $\mathrm{FA}_{\mathrm{C}}$ généralise le phénomène d'enchainement conjectural évoqué à l'instant, mais en le systématisant sous la forme d'un procédé narratif. Dans les Aventures de Tintin, le $\mathrm{FA}_{\mathrm{C}}$ énoncé par Tintin est en effet souvent employé pour situer un évènement qui n'est pas représenté dans la séquence d'images. Plus précisément, Tintin formule au $\mathrm{FA}_{\mathrm{C}}$ la reconstitution d'un segment de récit iconique qui fait défaut. L'exemple ci-dessous en est une expression typique :

Mais alors, j’y songe, le petit papier... se sera envolé lorsque je suis entré, la première fois, dans mon bureau, pour y prendre ma loupe !...

(CO7B2-B3, Tintin)

La séquence dont cet exemple est extrait couvre 23 cases, pratiquement deux planches complètes. Toutes les allées et venues de Tintin, entre son salon où il a posé le papier, et son bureau où il va chercher sa loupe, sont minutieusement détaillées ${ }^{22}$. Le seul évènement que le lecteur ne verra pas, car il n'est pas montré, est l'envol du petit papier sous l'effet du courant d'air.

Or ce courant d'air se reproduit un peu plus tard, et provoque le claquement de la porte qui effraie Tintin. De cela (« mais alors, j’y songe »), le jeune homme tire par abduction une conclusion empirique ( «le petit papier se sera envolé ») qui explique pourquoi il n’est plus sur la table du salon. Ce faisant, il remet virtuellement l'évènement à sa place dans la chaine narrative, ce qui reconstitue l'ordre de causalité des choses.

La fonction progressive ne consiste toutefois pas seulement à expliquer un fait au moyen d'une inférence sur sa cause, ce qui est le propre de la fonction régressive. À travers la relation de progression, le $\mathrm{FA}_{\mathrm{C}}$ réfère à une case manquante, il figure virtuellement une image de la séquence pour lui donner le statut de scène ellipsée. La situation montre toute l'importance du futur antérieur. Si l'énoncé était au passé composé ( « le petit papier s'est envolé lorsque je suis entré ») ou construit avec devoir épistémique (« le petit papier a dû s'envoler lorsque je suis entré »), la case virtuelle serait soit superflue (dans le cas de l'accompli), soit hasardeuse (dans le cas de l'incertain). Avec le $\mathrm{FA}_{\mathrm{C}}$, le contenu de la case est créé par la conjecture, en même temps que la case elle-même est réinstallée dans la séquence narrative.

Cette troisième partie a permis de cerner les rôles particuliers tenus par le $\mathrm{FA}_{\mathrm{C}}$ dans les Aventures de Tintin. Un rôle épistémologique d'une part, découlant directement de la valeur modale habituelle de ce temps verbal, mais qui prend une dimension particulière lorsque l'information modalisée a une contrepartie imagée dans la case. Un rôle diégétique d'autre part, associant subtilement la temporalité et la modalité du futur antérieur, afin qu'il intervienne de façon fonctionnelle dans la dynamique narrative du récit. L'importance du $\mathrm{FA}_{\mathrm{C}}$ dans les Aventures ne se mesure pourtant pas seulement à l'aune de ses fonctions. La deuxième caractéristique de ce temps verbal, aussi curieuse que précieuse, est d'orienter certains aspects référentiels de la relation texte-image.

\section{Le système référentiel du FAc dans Tintin}

À plusieurs reprises dans le point précédent, on a montré que les différentes fonctions du $\mathrm{FA}_{\mathrm{C}}$, en particulier lorsque celui-ci est manié par Tintin, étaient tournées vers la structuration logique, épistémique et chronologique du récit. La dernière partie de l'étude aborde maintenant une conséquence directe de cette typologie fonctionnelle, mais située dans le champ référentiel, et centrée uniquement sur Tintin. Il apparait en effet que Tintin ne fait pas qu'ordonnancer le récit, il lui arrive aussi de l'inventer.

Les $\mathrm{FA}_{\mathrm{C}}$ énoncés par Tintin peuvent être regroupés en trois modalités référentielles distinctes. Dans les trois cas, le sémantisme du temps verbal concerne la référence à l'évènement, référence activée au moyen de la RTI. Nous verrons donc successivement comment, à travers son activation par le $\mathrm{FA}_{\mathrm{C}}$, l'évènement est invoqué in absentia dans la case, comment il peut être (re)qualifié dans l'enchainement, et comment il est parfois évoqué in proesentia dans la séquence. 


\subsection{La scène invoquée}

L'analyse de la fonction appelée progressive a établi de quelle manière une scène s'inscrivant dans la chaine de causalité mais cependant ellipsée dans la séquence d'image pouvait être réinstallée à sa place par le $\mathrm{FA}_{\mathrm{C}}$. Il arrive cependant que la scène à laquelle Tintin fait allusion n'existe pas dans la séquence. Pour les besoins de la narration (pour la poursuite du récit, notamment), il invente ce qui s'est passé au lieu de le deviner. Le $\mathrm{FA}_{\mathrm{C}}$ déploie alors un élément essentiel de son sémantisme, évoqué en première partie, et qui tient dans sa force performative : l'explication, se proclamant seule possible en vertu de l'autorité du producteur du $\mathrm{FA}_{\mathrm{C}}$, déclare la vérification superflue ${ }^{23}$.

Le phénomène est peu fréquent mais très caractéristique de la position diégétique particulière de Tintin, «narrateur par procuration ». On en examinera un exemple significatif :

\section{Il sera monté dans une auto qui l'attendait ici.}

(AT10A4, Tintin)

Tintin et Milou fouillent le parc de Moulinsart alors que des évènements étranges viennent de s'y dérouler. Milou est sur une piste, mais à la sortie d'une haie les deux compagnons se retrouvent au bord de la route et Milou perd la trace.

La composition de cette case AT10A4 citée est très importante : Tintin est représenté dans l'angle inférieur gauche, il est montré de trois quarts (on ne voit que ses épaules et l'arrière de sa tête), il regarde la route qui s'ouvre devant lui et le village au loin, au-delà du virage. La nuit tombe, le ciel est bleu profond, les arbres, poteaux, panneaux et clôtures sont noirs, la route mouillée miroite légèrement. Il ne manque à l'image que l'auto dont il parle, et qu'il semble voir devant lui comme si elle était sur le point de s'enfuir.

C'est donc cette sorte de vision de Tintin qui tient lieu ici de représentation de l'évènement. Les différents éléments référentiels qui composent l'énoncé au $\mathrm{FA}_{\mathrm{C}}$ (les anaphoriques $i l$, le et qui ; le déictique $i c i$; l'antérieur sera monté ; l'indéfini une) fabriquent la scène dont Tintin a l'intuition, et qu'il nous impose comme étant ce qui a eu lieu. On voit que Tintin se replace dans le cadrage de la scène afin de mieux la reconstituer, et qu'il invoque en mots (monter dans une voiture) une action qui dans la logique de la $\mathrm{BD}$ aurait été montrée en image(s). Une RTI très particulière s'installe par conséquent, entre un énoncé verbal et son référent iconique absent. Cette relation, construite par le héros-locuteur à l'intérieur de la case, permet au narrateur de combler la case manquante en lui substituant un $\mathrm{FA}_{\mathrm{C}}$ qui, du même coup, attache la séquence de quête qui s'achève (Milou et Tintin dans le parc) à l'enquête qui commence. La voiture qui démarre, celle que Tintin nous dessine, c'est l'aventure qui se met en route.

\subsection{La scène (re)qualifiée}

Le deuxième type de modalité référentielle que nous examinons porte sur un groupe de cases, formant ou non une séquence complète, mais dont l'enchainement permet le déroulement d'une scène. Dans ces situations, la scène dessinée n'est pas seulement reprise par un énoncé au $\mathrm{FA}_{C}$, elle est également revue (révisée), comme si le texte devait proposer une nouvelle lecture de l'évènement antérieur.

L'exemple analysé est tiré de l'aventure du Sceptre d'Ottokar ${ }^{24}$. Tintin enquête sur un mystérieux restaurant syldave, le Klow. Il s'y rend pour déjeuner, et là, prétextant de vouloir passer aux toilettes, il monte à l'étage et écoute à une porte. Le patron du restaurant s'en rend compte et le rappelle à l'ordre en répétant l'indication qu'il lui avait déjà donnée : «Au fond du couloir, Monsieur... ». Tintin redescend l'escalier en déclarant :

\section{Excusez-moi : j’aurai mal compris...}

(SO5C3, Tintin)

Au mépris de tout principe de sincérité (le lecteur et le patron ont observé le manège) et de toute logique conjecturale (il sait qu'il n'a pas «mal compris »), Tintin fait passer sa présence dans l'escalier pour une erreur d'itinéraire. Nous constatons que l'énoncé «J'aurai mal compris » reprend toute la scène à la manière d'une reprise anaphorique et la reformule. De plus, la référence portée par le $\mathrm{FA}_{\mathrm{C}}$ fonctionne à la fois comme anaphore verbale au niveau langagier, et comme signal pragmatique au niveau métalangagier. L'explication suit d'ailleurs le principe de double communication : adressé par Tintin au protagoniste (le 
patron), le message au $\mathrm{FA}_{\mathrm{C}}$ se présente comme la seule justification possible de la méprise ; en revanche, le message adressé au lecteur est que la conjecture, puisqu'elle ne peut pas en être une, est une ruse.

L'énoncé au $\mathrm{FA}_{\mathrm{C}}$ qui clôt ce groupe de cases est donc particulièrement remarquable. D'abord parce qu'il procède à la requalification ${ }^{25}$ d'une scène située en amont; nous savions que le $\mathrm{FA}_{\mathrm{C}}$ avait cette faculté de remonter dans le temps, mais nous voyons maintenant qu'il peut également faire remonter la conjecture, autrement dit réécrire un évènement après coup. Ensuite parce qu'il exemplifie une situation dans laquelle le locuteur produit une conjecture qu'il sait fausse par avance. Cet exemple contredit d'ailleurs une contrainte, défendue par Dendale (2001: 4), prévoyant que «le locuteur pourrait difficilement présenter comme supposition une réalité qu'il est parfaitement bien placé pour connaitre de façon directe ». On vérifie du même coup que le récit en images (et sans doute la fiction en général), même s'il imite l'interaction langagière, n'obéit pas tout à fait aux mêmes règles ${ }^{26}$.

\subsection{La scène évoquée}

Lorsque Tintin reconstitue une scène sur la base d'une abduction, le verbe au $\mathrm{FA}_{\mathrm{C}}$ a pour fonction principale d'expliquer les faits, en pointant leur origine ou en formulant leur enchainement. Nous avons rencontré plusieurs de ces cas au fil de l'étude, dans lesquels Tintin devine ce qui s'est passé, par inférence ou conjecture forcée, et se fait par là le vecteur de l'information dont le lecteur a besoin.

Toutefois, dans plusieurs exemples de notre corpus (TA18B1, CP58C4, IN56C3, BC2A3, etc.), la valeur explicative de l'énoncé au $\mathrm{FA}_{\mathrm{C}}$ ne se limite pas à la modalité conjecturale du verbe conjugué, et se complète d'une RTI de type anaphorique qui catégorise ou dénomme les éléments nécessaires à la compréhension des faits. Le principe est toujours le même, à peu de choses près : l'énoncé renvoie anaphoriquement à un élément de la case, ou à la case elle-même (car dans ce cas la scène est toujours évoquée in prcesentia), mais la scène est montrée au lecteur d'une manière qui ne lui permet pas de la comprendre per se. L'exemple suivant illustre cette situation :

\section{Je comprends ! Ils auront essayé de briser les fils et ils auront provoqué un court- circuit!}

(IN28A2, Tintin)

L'action se déroule en trois cases :

a) Tintin, qui a ligoté deux bandits à un arbre à l'aide de câbles électriques, entend derrière lui ces mêmes bandits pousser des cris de douleur ;

b) Il se retourne et les voit s'enfuir ;

c) Il les poursuit en disant «Je comprends! Ils auront essayé de briser les fils et ils auront provoqué un court-circuit !».

L'exemple cité comporte donc non seulement l'explication de ce qui s'est passé, mais aussi l'élucidation des cris que les bandits ont poussés. En outre, le référent de la reprise anaphorique «provoquer un court-circuit» dans l'énoncé de Tintin s'identifie après coup à travers l'aspect et la typographie des phylactères, caractéristiques du choc électrique dans le code sémiotique de la BD. Les bandits se seront électrocutés.

On peut remarquer que l'explication donnée au $\mathrm{FA}_{\mathrm{C}}$ par Tintin («Ils auront provoqué un courtcircuit») fait de provoquer un court-circuit une anaphore résomptive ${ }^{27}$ qui reprend des informations graphiques et les condense sous la forme d'un syntagme propre à garantir la compréhension de l'incident. Autrement dit, l'interprétation que fait Tintin n'a pas pour objet la fuite des prisonniers (comment ils se sont libérés, par exemple), mais les cris qui se font entendre.

Il apparait ainsi que pour expliquer au lecteur-narrataire ce qu'il ne peut pas comprendre par lui-même (sauf à risquer de se tromper lourdement, chose non prévue dans le contrat narratif du récit d'aventures), Tintin dispose de deux armes importantes et complémentaires: le don de produire des conjectures toujours justes; le don de classer les constituants du monde (Meyer, 2003, 2005) et de nommer sans erreur les choses, faits et gens.

Un exemple pris dans l'aventure des Cigares du pharaon relève du même procédé :

\section{Ils auront dérapé dans le virage..}

(CP58C4, Tintin) 
Tintin est à la poursuite de deux hommes qui ont enlevé le fils du maharadjah. La scène se passe dans une route de montagne, les fuyards ont une voiture puissante et roulent dangereusement. Tintin, encore nettement distancé, aperçoit soudain au loin un panache de fumée noire. Lorsqu'il arrive sur les lieux, c'est pour constater que la voiture est tombée dans le ravin et déclarer : "Ils auront dérapé dans le virage $\gg$.

L'explication des faits ne réside donc pas seulement dans la conjecture en tant que telle (d'autant que Tintin a parlé trop vite, cf. la note 23), mais également dans le fait que le $\mathrm{FA}_{\mathrm{C}}$ porte sur un énoncé qui reprend en la conceptualisant une chaine d'évènements. Une image en case aurait pu représenter cela (ce n'est pas le cas), l'explication aurait pu être longue ou confuse... c'est tout le contraire qui se produit. Tintin, en très peu de mots parfaitement adéquats, résume les choses si clairement qu'il est presque impossible qu'elles aient pu se passer autrement.

L'énoncé au futur antérieur conjectural permet donc à Tintin d'agir à la fois sur le sens des évènements, en interprétant la valeur épistémique des informations ou des paroles échangées, et sur la référence des situations, en maitrisant, au sein même du récit iconotextuel, les relations texte-image de nature anaphorique. Tintin invente une sorte d'anaphore conjecturale.

\section{Conclusion}

Notre étude consacrée à la fréquence et la polyvalence exceptionnelles du futur antérieur conjectural dans les Aventures de Tintin s'est déroulée comme un parcours en trois étapes, chacune s'appliquant à analyser un rôle spécifique de ce temps verbal et de sa modalité particulière dans l'œuvre de Hergé.

La première étape, qui concerne le rôle épistémique du $\mathrm{FA}_{\mathrm{C}}$, a montré comment celui-ci partage les savoirs à l'intérieur de la scène narrative figurée. On a ainsi établi que la valeur conjecturale de l'énoncé se trouve soit renforcée soit contredite par l'information iconique, ce qui permet de hiérarchiser les différents protagonistes du récit en fonction de leur degré de connaissance de la situation énonciative, d'organiser la manière dont le lecteur récupère les informations dont il a besoin pour comprendre, et enfin de donner à Tintin une autorité narrative et explicative particulière.

La deuxième étape a défendu l'idée que le $\mathrm{FA}_{\mathrm{C}}$ assure également un rôle diégétique dans l'œuvre, rôle particulièrement important dans la chaine narrative du récit en images. Associé aux différentes formes de la RTI, notamment celles qui garantissent l'enchainement des cases et la progression du récit, le futur antérieur conjectural oriente la séquence de cases du point de vue de la logique causale, et organise la chronologie de l'évènement figuré en intervenant après coup sur sa progression.

Enfin, la troisième étape a fait émerger un rôle sémantique du $F A_{C}$, plus précisément une dimension référentielle qui vient compléter la référence du texte et de l'image en conjonction. Cette dernière étape a permis de montrer que la relation explicative ou interprétative portée par le $\mathrm{FA}_{\mathrm{C}}$ véhicule des formes d'anaphore texte-image de type conceptuel. Les contenus iconiques sont repris sous la forme d'énoncés conjecturaux qui les verbalisent, afin d'assurer ou faciliter la compréhension de l'évènement.

Ces trois fonctions du $F A_{C}$, épistémique, diégétique et sémantique, sont étroitement liées au personnage de Tintin, qu'elles installent et affirment dans sa position omnipotente et omnisciente. À n'en pas douter, le futur antérieur conjectural est le temps de Tintin : comme temps verbal il accompagne son rôle de maitre du jeu et des évènements ; comme catégorie modale il légitime son type de raisonnement et le hisse au rang de modèle narratif. 


\section{Références bibliographiques}

Bellahsène, L., (2007), "L'expression de la conjecture: le cas du futur en français », In Bouchard, D., Représentation du sens linguistique, Bruxelles : De Boeck Université, coll. « Champs linguistiques », p. 253-266.

Celle, A., (2010), «Personne et modalité : modes d'accès à l'autre », In Lozano, J. V., et al., (éds), Les représentations linguistiques de la personne, Rouen : ERIAC-Université de Rouen, p. 277-290.

Dendale, P., (2001), "Le futur conjectural versus "devoir" épistémique : différences de valeur et de restrictions d'emploi », Le français moderne, t. LXIX, 1, Paris : CILF, p. 1-20.

Dendale, P., De Mulder, W., (1996), «Déduction ou abduction: le cas de devoir inférentiel », In Guentchéva, Z., (éd.), L'énonciation médiatisée, Louvain : Peeters, p. 305-318.

Desclés, J.-P., Guentchéva, Z., (2001), "La notion d'abduction et le verbe devoir "épistémique" », Cahiers Chronos, 8, Amsterdam : Rodopi, p. 103-122.

Gosselin, L., (2010), Les modalités en français : la validation des représentations, Amsterdam : Rodopi, coll. «Études Chronos ».

Grevisse, M., Goossse, A., (2007), Le bon usage, Bruxelles : De Boeck. (Première édition : 1936.)

Groensteen, T., (1999), Système de la bande dessinée, Paris : PUF, coll. « Formes sémiotiques ».

Haillet, P. P., (2007), Pour une linguistique des représentations discursives, Bruxelles : De Boeck.

Lacan, J., (1966), Écrits, Paris : Seuil, coll. « Le champs freudien ».

Martin, R., (1992 [1983]), Pour une logique du sens, Paris : PUF, coll. « Linguistique nouvelle».

Meyer, J.-P., (2002), «Tintin et le prototype, ou Quand les singes auront des ailes », In Kleiber, G., Le Querler, N., (éds.), Traits d'union, Caen : Presses Universitaires de Caen, p. 193-207.

Meyer, J.-P., (2003), «Le Temps dessiné : Sémantisme de la référence temporelle dans le récit en images », Cahiers Chronos, 11, Amsterdam : Éditions Rodopi, p. 151-172.

Meyer, J.-P., (2005), «Reflets et écrans dans les Aventures de Tintin: le récit au miroir déformant de l'image », Creliana, 4-5, Mulhouse : Éditions du CREL, p. 93-102.

Meyer, J.-P., (2008), « Temps et narration dans la bande dessinée : l'invention de la temporalité dans les aventures de Little Nemo in Slumberland», Entr'actes : regards croisés en Sciences humaines, Strasbourg: Éditions de l'université Marc-Bloch, p. 17-31.

Meyer-Bolzinger, D., (2012), La méthode de Sherlock Holmes. De la clinique à la critique, Paris : Campagne Première.

Morency, P., (2010), «L'enrichissement épistémique du futur », Cahiers Chronos, 21, Amsterdam : Rodopi, p. 197214.

Peeters, B., (1998), Case, planche, récit. Lire la bande dessinée, Tournai : Casterman.

Riegel, M., Pellat, J.-C., Rioul, R., (2009), Grammaire méthodique du français, Paris : PUF, coll. "Quadrige». (Première édition : 1994.)

Steinmeyer, G., (1987), «Le futur antérieur comme temps du passé : remarques sur un emploi particulier fréquent du futur antérieur en français ", International Review of Applied Linguistics in Language Teaching, vol. 25, 1-4, Berlin : De Gruyter, p. 119-130.

Sthioul, B., (1998), «Temps verbaux et point de vue », In Moeschler, J., dir., Le temps des évènements. Pragmatique de la référence temporelle, Paris : Kimé, p. 197-220.

Taji, K., (2003), « À propos du futur antérieur », L'Information grammaticale, 97, Paris : Société pour l'information grammaticale, p. 37-40.

Wagner, R.-L., Pinchon, J., (1978), Grammaire du français classique et moderne, Paris : Hachette. (Première édition : 1962.)

Weinrich, H., (1973, [1964]), Le Temps, Paris : Seuil, coll. « Poétique ».

Wilmet, M., (1976), Études de morphosyntaxe verbale, Paris: Klincksieck, coll. «Bibliothèque française et romane $»$.

Wilmet, M., (1997), Grammaire critique du français, Bruxelles : Duculot. 


\section{Annexe 1 : Tableau de répartition des occurrences de Futur antérieur}

\begin{tabular}{|c|c|c|c|c|c|c|}
\hline & & & & FA Modal & & \\
\hline & & Temporel & $\begin{array}{c}\text { Marqueur } \\
\text { lexical }\end{array}$ & \begin{tabular}{|c|} 
Marqueur \\
syntaxique
\end{tabular} & \begin{tabular}{|c|} 
Pas de \\
marqueur
\end{tabular} & Total \\
\hline PS & Au pays des Soviets (1930) & 3 & 0 & 0 & 0 & 3 \\
\hline $\mathrm{TC}$ & Tintin au Congo (1931) & 0 & 0 & 0 & 0 & 0 \\
\hline TA & Tintin en Amérique (1932) & 3 & 1 & 0 & 2 & 6 \\
\hline $\mathrm{CP}$ & Les Cigares du pharaon (1934) & 2 & 1 & 0 & 1 & 4 \\
\hline LB & Le Lotus bleu (1936) & 1 & 0 & 1 & 4 & 6 \\
\hline $\mathrm{OC}$ & L’Oreille cassée (1937) & 1 & 0 & 0 & 0 & 1 \\
\hline $\mathrm{IN}$ & L'Ile noire (1938) & 1 & 0 & 0 & 6 & 7 \\
\hline SO & Le Sceptre d'Ottokar (1939) & 2 & 0 & 0 & 2 & 4 \\
\hline $\mathrm{CO}$ & Le Crabe aux pinces d'or (1941) & 1 & 0 & 0 & 4 & 5 \\
\hline EM & L’Étoile mystérieuse (1942) & 1 & 0 & 0 & 0 & 1 \\
\hline SL & Le Secret de La Licorne (1943) & 0 & 2 & 0 & 4 & 6 \\
\hline TR & Le Trésor de Rackham le rouge (1944) & 1 & 0 & 0 & 2 & 3 \\
\hline SB & Les 7 boules de cristal (1948) & 0 & 0 & 0 & 0 & 0 \\
\hline TS & Le Temple du Soleil (1949) & 0 & 0 & 0 & 1 & 1 \\
\hline $\mathrm{PO}$ & Au Pays de l'or noir (1950) & 0 & 0 & 0 & 0 & 0 \\
\hline $\mathrm{OL}$ & Objectif Lune (1953) & 0 & 1 & 2 & 0 & 3 \\
\hline ML & On a marché sur la Lune (1954) & 0 & 0 & 0 & 1 & 1 \\
\hline AT & L'Affaire Tournesol (1956) & 0 & 0 & 0 & 2 & 2 \\
\hline CS & Coke en stock (1958) & 1 & 0 & 0 & 2 & 3 \\
\hline TT & Tintin au Tibet (1960) & 0 & 0 & 0 & 1 & 1 \\
\hline $\mathrm{BC}$ & Les Bijoux de la Castafiore (1963) & 2 & 1 & 1 & 3 & 7 \\
\hline VS & Vol 714 pour Sydney (1968) & 0 & 0 & 0 & 0 & 0 \\
\hline TP & Tintin et les Picaros (1976) & 4 & 1 & 0 & 2 & 7 \\
\hline & & 23 & 7 & 4 & 37 & 71 \\
\hline & Total & FA & $\begin{array}{c}\text { Marqueur } \\
\text { lexical }\end{array}$ & $\begin{array}{c}\text { Marqueur } \\
\text { syntaxique }\end{array}$ & $\begin{array}{c}\text { Pas de } \\
\text { marqueur }\end{array}$ & Total \\
\hline & & & & FA Modal & & \\
\hline
\end{tabular}

\section{Annexe 2 : Corpus des occurrences de futur antérieur dans les Aventures de Tintin}

Au pays des Soviets

- 11A $\mathrm{Hi}$ ! Hi ! Il n'aura pas été loin. (Un policier allemand)

- 51A Non, mais, quand vous aurez fini de me secouer comme un prunier! (Tintin)

- 71B2 La barre de fer n'aura pas été chauffée en vain. (Tintin)

Tintin en Amérique

- 13B2 Le contact de l'eau l'aura ranimé. (Bobby Smiles) 
-18B1 On l'aura prévenu de mon arrivée... (Tintin)

-36C1-C2 Il ne sera pas dit que le shérif aura... vite encore un dernier verre : le dernier... aura laissé lyncher un innocent... (Shérif ivre de la petite ville)

- 40A2 Mais, bien entendu, j'aurai eu soin de vous attacher sur la voie... (Bobby Smiles)

- 51D2 Un des bandits, là-haut, aura surement donné l'alarme. (Tintin)

Les Cigares du pharaon

- 5D2 Ils seront probablement partis pour le Caire. (Lettre du chef de la secte Kih-Oskh)

- 24C2 Il avait l'air épuisé. Nous l'aurons vite rejoint. (Dupond)

- 48A3 Il ne peut pas avoir une grande avance sur nous : nous l'aurons vite rejoint. (Dupond)

-58C4 Ils auront dérapé dans le virage... (Tintin)

Le Lotus Bleu

-3C3 Plus personne... Il aura filé sans demander son reste... (Tintin)

- 12B3 Le thé !... Il aura bu du thé qui était répandu à terre !... (Tintin)

- 37C1 Et demain, à l'aube, Tintin aura terminé sa carrière... car je ne vois plus comment je pourrais m'en tirer... (Tintin)

-46C4 Mon Dieu ! je l'aurai mal remis en poche après l'avoir montré à Tintin !... (Dupond)

- 51A2 À mon avis, il sera descendu en cours de route... (Richards, inspecteur de Dawson)

- 55C2 Non, il se sera méfié... (Serviteur du Lotus Bleu)

L'Oreille cassée

- 25C2 Dès qu'il nous aura révélé l'endroit où se trouve le fétiche... (Alonzo Perez)

L'Ile noire

-27A4 Ah! Je comprends! L'imbécile aura marché sur ce râteau. (Ivan)

- 28A2 Je comprends! Ils auront essayé de briser les fils et ils auront provoqué un court-circuit! (Tintin)

-38C1 Nous les aurons vite rejoints. (Pilote de l'avion à la poursuite de Müller et Ivan)

- 51B1 Je parie que c'est Tintin qui a fait le coup : il se sera enfui quand il nous aura entendus. (Müller)

- 56C3 Ça va mal! Il aura été délivrer tous les autres : je vais avoir toute la bande sur le dos! (Tintin)

Le Sceptre d'Ottokar

- 5C3 Excusez-moi : j'aurai mal compris... (Tintin)

- 43B1 Parce que le voleur aura lancé le sceptre par-dessus la muraille qui entoure le château (Dupont)

- 49B2 Ils n'ont pas une grande avance sur nous... Nous les aurons vite rejoints... (Tintin)

- 49D2 Dans une demi-heure nous aurons quitté la Syldavie et le sceptre sera en sureté !... (Conspirateur)

Le Crabe aux pinces d'or

- 7B2-B3 Mais alors, j'y songe, le petit papier...se sera envolé lorsque je suis entré, la première fois, dans mon bureau, pour y prendre ma loupe !... (Tintin)

-50D2 Mon pied aura heurté un de ces mauvais pavés... (Allan)

- 55D3 S'il fait le méchant, j'aurai vite réglé son compte... (Allan)

- 58C3 Il aura fui par l'autre issue! (Tintin)

- 61C3 Oui, on l'aura fait disparaitre! (Tintin)

L'Étoile mystérieuse

- 20C2 Chers auditeurs, dans quelques minutes, l'Aurore nous aura quittés et voguera vers le Nord, vers les régions arctiques. [...] (Présentateur de radio au départ de l'expédition)

Le Secret de la Licorne

-2A4 Tu l'auras sans doute oublié à la maison... (Dupond)

-12A1 C'est ça!... J'y suis !... Ce papier devait se trouver enroulé dans le mât de mon bateau. Il s'en sera échappé lorsque le mât s'est détaché, en tombant, et il aura roulé sous le meuble... (Tintin)

- 27B2 Volé ? Vous l'aurez sans doute oublié chez vous. (Haddock)

- 41D1 Oh! il n'aura pas eu de difficultés à se cacher. (Maxime Loiseau)

- 51A1 Il aura pris la fuite, cet imbécile. (Maxime Loiseau)

Le Trésor de Rackham le rouge

- 57A3 Maxime Loiseau aura su que nous étions à bord et il n'aura rien osé entreprendre contre vous... (Dupond)

- 57C3 ...et quand vous aurez fini de concasser l'avoine, vous pourrez vous mettre au hache-paille... (fermier ami des Dupondt)

Le Temple du soleil

-1C1 Vous aurez mal vu... (le chef de la police de Callao)

Objectif Lune

-3B4 Je me demande si Tournesol sera venu nous attendre... (Tintin)

- 26C1 Ce ne sera pas commode, je le crains !... Une fois son coup fait, le gaillard va chercher à se faire oublier... Quant à savoir quels sont les documents qu'il a remis à ses complices, je suis persuadé qu'il n'aura pas commis l'imprudence de voler les originaux, ce qui nous aurait permis de localiser les recherches. (Tintin) 
- 26C2 (suite) À mon avis, il en aura tout simplement pris copie... Si je n'avais pas été là, cette nuit, le forban aurait tranquillement remis ces pièces à son complice et jamais personne ne se serait douté de la chose... (Tintin)

On a marché sur la Lune

- 58B4 Il aura de nouveau perdu connaissance... (Baxter)

L'Affaire Tournesol

- 10A4 Il sera monté dans une auto qui l'attendait ici. Inutile d'insister; viens, nous allons rejoindre les autres. (Tintin)

- 28C1-C2 ...Voilà comment je reconstitue l'affaire. [...] Dans une lettre, notre ami décrit ses travaux à Topolino. Cette lettre aura été surprise par le domestique de Topolino, un Bordure nommé Boris, qui alerte les services secrets de son pays. Mais l'espionnage syldave a vent, lui aussi, de l'invention et envoie à Moulinsart un de ses agents. Celui-ci tombe là sur un rival bordure, qui le blesse. (Tintin)

Coke en stock

-33C1 Oui... Malheureusement, ils auront fait rapport, et dans ce cas... (Tintin)

- 51D4 Dans quelques heures, le « Ramona » aura disparu corps et biens... (Rastapopoulos)

- 61D4 Nestor se sera fait du mauvais sang pour d'innocentes gamineries... (Haddock)

Tintin au Tibet

- 49D2 Tel aura été le sort de ton ami Tchang, et jamais, jamais tu n'en retrouveras la moindre trace... (Grand Précieux)

Les Bijoux de la Castafiore

- 2A3 Elle se sera éloignée du campement que nous venons de voir... (Tintin)

- 13B2 Moi, je vous aurai mis en garde. (Commandant de gendarmerie)

-16D3 Ça, c'est le docteur qui s'en va : il sera venu pour le plâtre du capitaine... Mais à qui, diable! est cette autre voiture ?... (Tintin)

- 43D2 Ne vous dérangez pas : elle les aura retrouvés dans cinq minutes. (Haddock)

- 44C3 Peut-être le bijou sera-t-il tombé à terre, et... (Tintin)

- 49B2 Oh! désolé... Un projecteur qui aura sauté... (Tournesol)

- 62A2 Boullu vous l'aura dit, je suppose... (Haddock)

Tintin et les Picaros

-16D3 Grippé !... Il se sera méfié, oui !... Mais il faut absolument qu'il vienne... Et d'ailleurs, comme je le connais, il viendra de toute façon !... (Sponsz/Esponja)

-24B3 Version officielle : les Picaros auront tenté de vous enlever !... (Pablo)

- 36D4 C'est probablement un caïman que tu auras vu... (Pilote de l'hélicoptère)

- 54A3 Et puis, d'ailleurs, je saurai magnifiquement leur prouver ma reconnaissance : ils seront tous décorés de l'ordre de San Fernando, dès que j'aurai triomphé de cet infâme Tapioca !... (Alcazar)

- 54B1 (suite) Demain, dans l'après-midi, nous arriverons à Tapiocapolis (qui s'appellera bientôt Alcazaropolis!), pour la première journée du carnaval. Avant d'entrer en ville, nous aurons minutieusement mis au point notre plan d'action... (Alcazar)

- 54B2 (suite et fin) Nous aurons revêtu les costumes des Turlurons et nos armes seront à portée de la main... (Alcazar)

1. La rédaction de ce texte est conforme aux Rectifications de l'orthographe adoptées par l'Académie française (J.O. du 6 décembre 1990).

2. Avec plus de poésie, Taji (2003: 40) explique que le futur antérieur conjectural est fait «pour réserver ou atténuer notre jugement définitif sur un évènement passé » lorsque "nous nous en remettons à l'avenir [...] en attendant sa confirmation ultérieure ».

3. Toutes les références aux albums de Tintin renvoient à l'édition historique Casterman. Chaque album est codé en deux lettres (ici CS pour Coke en stock, voir Annexe 1 infra), puis vient le numéro de la page puis le code de la vignette (A, B, C pour les rangées de cases ; 1, 2, 3 pour le numéro d'ordre dans la rangée.)

4. Pour Wilmet, l'évènement antérieur est enregistré « sous bénéfice d'inventaire. » (1997:380).

5. L'énoncé, ne pouvant être vérifié au moment de l'énonciation, exprime une vérité annoncée comme différée. (cf. Dendale, 2001).

6. Voir Riegel et al., (2009 : 554), mais aussi Steinmeyer, (1987).

7. D'après Weinrich (1989: 158), ce qui caractérise le mieux le futur antérieur conjectural est l'incertitude.

8. Voir Dendale $(2001: 2)$ pour une discussion à ce sujet. 
9. C'est pour cette raison que Wilmet (1976:41-60) ne reconnait pas tout à fait l'appellation conjectural. Classant lui aussi les formes épistémiques du futur antérieur dans les emplois «à sens de passé composé », il considère leur valeur aspectuelle comme « restrictive »: le locuteur « refuse d'entériner l'évènement passé ou son résultat présent » (58).

10. Dendale (2001: 15) rappelle à ce propos que «la conclusion qui est formulée, si elle a été obtenue de façon rapide, est avancée très souvent de façon très assurée par le locuteur - peut-être justement à cause de cela - et parait alors se fonder sur une grande confiance - vraie ou feinte - en ce qu'il dit. »

11. Meyer-Bolzinger (2012, 168-169) décrit le récit d'enquête comme une métanarration, dans laquelle résoudre une énigme devient raconter une histoire.

12. Voir en particulier Desclés \& Guentchéva, (2001 : 104-106) pour une démonstration très complète, mais aussi la discussion qu'en propose Gosselin, (2010:450).

13. Voir l'analyse de Gosselin, (2010: 451), que nous partageons sur ce point : la plausibilité, en tant que jugement global porté sur le raisonnement ou sa conclusion, est un cadre discursif plutôt qu'une prémisse du raisonnement. Dans le genre narratif qui nous intéresse ici, celui des récits de quête ou d'enquête, c'est bien ce cadre discursif annoncé comme tel qui autorise l'inférence abductive.

14. Lacan insiste sur ce point, dans «Situation de la psychanalyse et formation du psychanalyste en 1956 » (Écrits, 1966, 459-491) : «La conjecture n'est pas l'improbable : la stratégie peut l'ordonner en certitude » (472).

15. Voir le corpus complet en Annexe 2, infra.

16. Plusieurs temps verbaux, en particulier l'imparfait, jouent ce rôle dans Tintin et dans la BD. Voir Meyer, (2003) et Meyer, (2008).

17. N'oublions que Haddock et Milou le comparent plusieurs fois à Sherlock Holmes.

18. Ce que Morency (2010 : 209) appelle « les expressions facilitant la lecture épistémique ».

19. Les cas où l'image donne raison au personnage sont rares, pour ne pas dire exceptionnels. On ne repère que deux exemples : dans L'Ile noire, (IN27A4, Ivan) : "L'imbécile aura marché sur ce râteau », et dans On a marché sur la Lune, (ML58B4, Baxter) : «Il aura de nouveau perdu connaissance ». Ce dernier exemple est analysé au point 3.2.2. 20. En contrepartie, le récit garantit l'hypothèse conjecturée et permet à l'enquêteur de ne pas se tromper. C'est l'un des secrets de l'abduction holmésienne. Voir à ce sujet Meyer-Bolzinger, op. cit., (36).

21. Pour reprendre la typologie des statuts de l'information, on a là un cas - peu fréquent - ou $S_{L}>S_{P}$ mais où la conjecture de $\mathrm{P}$ est vraie.

22. Le fameux «petit papier » met Tintin sens dessus-dessous : dans un état de distraction et de confusion extrême, il gronde Milou parce qu'il ronge un os, puis jette la loupe à la poubelle au lieu de jeter l'os, confond la loupe et l'os, s'effraie d'une porte qui claque, égare ses affaires...

23. Il n'existe qu'un seul cas d' «erreur» de Tintin dans cette situation. À la fin de l'aventure des Cigares du pharaon, Tintin aperçoit dans un précipice la voiture des bandits qu'il poursuivait, en flammes, et déclare : "Ils auront dérapé dans le virage... » (CP58C4, Tintin). Mais assez rapidement il se ravise et revient sur ce scénario trop rapidement invoqué, comme s'il s'apercevait lui-même qu'il n'a pas assez d'éléments pour le conjecturer.

24. Plusieurs autres exemples peuvent être cités, pas tous dus à Tintin d'ailleurs : "Ça va mal! Il aura été délivrer tous les autres : je vais avoir toute la bande sur le dos! » (IN56C3, Tintin); "Vous aurez mal vu... » (TS1C1, Chef de la police de Callao); «Oui... Malheureusement, ils auront fait rapport, et dans ce cas... » (CS33C1, Tintin); «Version officielle : les Picaros auront tenté de vous enlever !... » (24B3, Pablo).

25. Parmi les exemples donnés dans la note précédente, certains sont descendants, c'est-à-dire que la scène référée suit le $\mathrm{FA}_{\mathrm{C}}$ qui la qualifie. D'autres au contraire sont remontants, à l'instar de l'exemple analysé, où c'est le $\mathrm{FA}_{\mathrm{C}}$ qui suit la scène référée.

26. Sur ce sujet, voir également Celle (2010), qui examine la situation en la comparant à l'anglais.

27. L'anaphore résomptive, également appelée conceptuelle, est une forme particulière d'anaphore infidèle. Elle permet de reprendre une grande quantité d'information, sous la forme d'un nom qui la condense et la conceptualise. Voir Riegel, et al., (2009 : 1038). 